\title{
Is colorectal cancer screening necessary before 50 years of age?
}

\author{
Yoon Suk Jung \\ Division of Gastroenterology, Department of Internal Medicine, Kangbuk Samsung Hospital, Sungkyunkwan University School of Medicine, \\ Seoul, Korea
}

Colorectal cancer (CRC) is the third most common cancer and the fourth most common cause of death from cancer worldwide. The current guidelines recommend that screening of individuals at an average risk for CRC be started at the age of 50 years; as a result, the incidence and mortality rate of CRC have decreased in adults above the age of 50 years over the past few decades. ${ }^{2}$ In contrast, the incidence in adults aged below 50 years has increased. ${ }^{2}$ Bailey et al. ${ }^{3}$ demonstrated that the annual incidence of metastatic CRC as well as that of localized CRC has increased in young adults. They predicted that by 2030, the incidence of CRC would increase by $27.7 \%$ to $46.0 \%$ in individuals aged 35 to 49 years. ${ }^{3}$ Korean population-based data also show that the incidence of CRC increased by $78 \%, 63 \%, 53 \%$, and $50 \%$ in individuals aged 30-34, 35-39, 40-44, and 45-49 years, respectively, between 1999 and 2013. ${ }^{4}$ Further, younger patients with CRC have been reported to tend to have more aggressive disease than that observed in older patients, with worse outcomes. ${ }^{2} \mathrm{~A}$ study by Riaz et al. ${ }^{5}$ also showed that the majority of patients with young-onset CRC had advanced stage of the disease. Considering that adults aged below 50 years comprise the bulk of the economically active population, an increase in CRC in this demographic may confer a socioeconomic burden in the future. Therefore, clinicians should be concerned

Received August 20, 2017. Revised August 22, 2017.

Accepted August 22, 2017

Correspondence to Yoon Suk Jung, Division of Gastroenterology,

Department of Internal Medicine, Kangbuk Samsung Hospital,

Sungkyunkwan University School of Medicine, 29 Saemunan-ro, Jongno-gu,

Seoul 03181, Korea. Tel: +82-2-2001-8577, Fax: +82-2-2001-2049, E-mail:

ys810.jung@samsung.com

Financial support: None. Conflict of interest: None. about young-onset advanced colorectal neoplasia (ACRN), including CRC.

I carefully read the study by Riaz et al..$^{5}$ (Red flag symptoms: detailed account of clinicopathological features in young-onset colorectal cancer. Intest Res 2017;15:203-207). The study investigated the clinicopathological features of young-onset CRC (patients aged below 50 years) in Pakistan. ${ }^{5}$ Unlike previous studies, in this study, the authors found that the majority of patients with young-onset CRC had no family history of CRC. In young patients, a family history of CRC may be less accurate, because their parents may have not lived long enough to realize their potential of CRC. This may be one of the reasons why a family history of CRC was not associated with young-onset CRC in their study.

The study by Riaz et al. ${ }^{5}$ showed that most patients with young-onset CRC had a left-sided tumor, and most patients with a left-sided tumor developed rectal bleeding. The authors suggested that painless rectal bleeding in young individuals should alert physicians for appropriate investigation, as the majority of patients with young-onset CRC develop painless bleeding 2 to 3 years before the appearance of other symptoms. However, in the study by Riaz et al., only a small proportion of patients with young-onset CRC were at TNM stage 1 ( $7.6 \%)$ and $2(16.2 \%)$, whereas most of the patients were at stage $4(40.0 \%)$, followed by stage $3(36.2 \%)$. Given that the majority of patients with young-onset CRC were at an advanced stage of the disease, it may be too late to perform endoscopy after the occurrence of symptoms of rectal bleeding. Hence, it is necessary to find young individuals who are expected to have a high risk of CRC.

As part of these efforts, several recent studies have examined the risk factors for ACRN in young individuals aged be-

\footnotetext{
๑ Copyright 2017. Korean Association for the Study of Intestinal Diseases. All rights reserved.

This is an Open Access article distributed under the terms of the Creative Commons Attribution Non-Commercial License (http://creativecommons.org/licenses/by-nc/4.0)

which permits unrestricted non-commercial use, distribution, and reproduction in any medium, provided the original work is properly cited.
} 
low 50 years. ${ }^{6,7}$ The studies have shown that male sex, smoking, metabolic syndrome, and obesity are the risk factors for ACRN in young individuals, and suggested that individuals with such risk factors may benefit from starting screening colonoscopies before the age of 50 years. ${ }^{6,7}$

However, the most important concern with screening colonoscopy performed before the age of 50 years is its low costeffectiveness, because the risk of CRC or ACRN is relatively low in this population. ${ }^{2,6}$ To improve the cost-effectiveness of CRC screening in young population, several alternative CRC detection tests including sigmoidoscopy, fecal immunochemical test (FIT), and clinical risk stratification models may have to be considered. In particular, FIT may be a good alternative strategy to primary screening with colonoscopy owing to the advantages of non-invasiveness and low cost. A recent study demonstrating that the diagnostic performance of FIT for ACRN in a young population ( $<50$ years) was not inferior to that in the current screening-age population $(\geq 50$ years) supported that FIT may be a good test for detecting ACRN in a young population. ${ }^{8}$

Clinical risk stratification may also be useful in selecting young individuals at a high risk for ACRN. Clinical risk factors for ACRN (such as sex, smoking, family history of CRC, and obesity) can be identified easily in a clinical situation. However, although a few studies determined the risk factors for ACRN in young individuals aged less than 50 years, ${ }^{6,7}$ no study has yet established a risk-stratification model of ACRN that is reliable and applicable to such individuals. ${ }^{6,7}$ Several models that stratify the risk of ACRN have been developed to make CRC screening more effective. ${ }^{9,10}$ For instance, the Asia-Pacific Colorectal Screening (APCS) score and the Korean Colorectal Screening (KCS) score, which predict ACRN, were recently developed on the basis of the following risk factors: age, sex, family history of CRC, and smoking habits; the KCS scoring model also includes obesity. ${ }^{9,10}$ However, all these scoring models focused on individuals aged 50 years or older. For example, in the APCS and KCS scoring models, all individuals aged less than 50 years are given an age-score of zero points; hence, risk stratification in this population is limited. Future studies are warranted to develop a risk stratification model for ACRN that focuses on young individuals aged below 50 years.

A combination of strategies of clinical risk stratification and FIT results may be helpful for the detection of ACRN in young individuals. More specifically, a strategy where indi- viduals with a high clinical risk of ACRN would undergo an FIT, and then those with positive FIT results would undergo a confirmative colonoscopy may be a reasonable method of CRC screening in a young population. Further research is warranted to determine the best strategy for CRC screening in young population, and the cost-effectiveness of CRC screening before the age of 50 years.

\section{REFERENCES}

1. Seo JY, Choi SH, Chun J, et al. Characteristics and outcomes of endoscopically resected colorectal cancers that arose from sessile serrated adenomas and traditional serrated adenomas. Intest Res 2016;14:270-279.

2. Inra JA, Syngal S. Colorectal cancer in young adults. Dig Dis Sci 2015;60:722-733.

3. Bailey CE, Hu CY, You YN, et al. Increasing disparities in the age-related incidences of colon and rectal cancers in the United States, 1975-2010. JAMA Surg 2015;150:17-22.

4. Cancer incident cases and incidence rates by site (24 items), sex, age group. Korean Statistical Information Service Web site. http://kosis.kr/statHtml/statHtml.do?orgId= $117 \&$ tblId=DT_117N_A00023\&conn_path=I2\&language=en. Accessed August 15, 2017.

5. Riaz R, Masood N, Benish A. Red flag symptoms: detailed account of clinicopathological features in young-onset colorectal cancer. Intest Res 2017;15:203-207.

6. Jung YS, Ryu S, Chang Y, et al. Risk factors for colorectal neoplasia in persons aged 30 to 39 years and 40 to 49 years. Gastrointest Endosc 2015;81:637-645.e7.

7. Chang LC, Wu MS, Tu CH, Lee YC, Shun CT, Chiu HM. Metabolic syndrome and smoking may justify earlier colorectal cancer screening in men. Gastrointest Endosc 2014;79:961-969.

8. Kim NH, Park JH, Park DI, Sohn CI, Choi K, Jung YS. The fecal immunochemical test has high accuracy for detecting advanced colorectal neoplasia before age 50. Dig Liver Dis 2017;49:557-561.

9. Yeoh KG, Ho KY, Chiu HM, et al. The Asia-Pacific Colorectal Screening score: a validated tool that stratifies risk for colorectal advanced neoplasia in asymptomatic Asian subjects. Gut 2011;60:1236-1241.

10. Kim DH, Cha JM, Shin HP, Joo KR, Lee JI, Park DI. Development and validation of a risk stratification-based screening model for predicting colorectal advanced neoplasia in Korea. J Clin Gastroenterol 2015;49:41-49. 